http://jmscr.igmpublication.org/home/

ISSN (e)-2347-176x ISSN (p) 2455-0450

crossref DOI: https://dx.doi.org/10.18535/jmscr/v7i11.172

Journal Of Medical Science And Clinical Research

\title{
Jhajhria Infundibular Resection Adequacy Assessment Technique (JIRAAT) to assess the adequacy of Right ventricular outflow tract muscle bands resection
}

\author{
Authors \\ Dr Mohd Javed Banday, Dr Dhananjay K Bansal, Dr Surendra VVBS Chauhan, \\ Dr Narender Singh Jhajhria*, Dr Vijay Gupta, Dr Vijay Grover \\ ABVIMS and Dr R.M.L. hospital, New Delhi, New Delhi, Pin Code: 110001 \\ *Corresponding Author \\ Dr Narender Singh Jhajhria \\ Prof Dept of CTVS, Dr RML Hospital, New Delhi, India
}

\begin{abstract}
Tetrology of Fallot (TOF) and related conditions like Double Chambered Right Ventricle(DCRV), Double Outlet Right Ventricle (DORV) and Ventricular Septal Defect with infundibular Pulmonary Stenosis (VSD $P S)$ are increasingly being repaired through transatrial route because of poor long term results associated with right ventricular approach like increased incidence of arrhythmias and right ventricular dysfunction with the later approach. We have been almost exclusively using transatrial approach since last five years and have modified it a bit. We describe our modification in the intraoperative technique for assessing adequacy of infundiblar muscle resection on the cardioplegic arrested heart, and have named the modification as Jhajhria Infundibular Resection Adequacy Assessment Technique (JIRAAT). The advantages of such a technique are also discussed.

Keywords: Intracardiac repair, tetralogy of Fallot, TOF, transatrial repair, RVOT, JIRAAT.
\end{abstract}

\section{Introduction}

Transventricular approach has been in use since years for resection of Right Ventricle Outflow Tract (RVOT) in repair of TOF and related conditions. Closure of RVOT is done using pericardial patch. Besides patch related complications like calcification, there had been other concerns like the resultant scar which increases the incidence of ventricular arrythmias and impair the right ventricular function and can be cause of sudden death ${ }^{[1,2]}$.
Transatrial repair of TOF first reported in 1963 by Hudspeth et $\mathrm{al}^{[3]}$ had eliminated the complications of ventriculotomy and had gained popularity since last two decades. We describe our modification in the intraoperative technique for assessing adequacy of infundiblar muscle resection through transatrial technique on the cardioplegic arrested heart, and have named the modification as Jhajhria Infundibular Resection Adequacy Assessment Technique (JIRAAT). The advantages of our technique are also discussed. 


\section{Patients}

We have used this technique so far in more than 138 patients, 120 of them were of TOF, 2 were DCRV, 8 were DORV and 8 patients were of VSD PS. Informed consent from the parents of the patients was obtained.

\section{Surgical Technique}

A uniform operative technique was used with aorto-bicaval cannulation for cardiopulmonary bypass and moderate systemic hypothermia $\left(28^{\circ} \mathrm{C}\right.$ nasopharyngeal temperature). Ductus arteriosus dissected and ligated if present. Myocardial protection was achieved with delNido cold blood cardioplegia delivered through aortic root supplemented with ice slush. Following the cardioplegic arrest, the right atrium was opened by an incision starting from the base of the right atrial appendage and extending to the medial aspect of the inferior vena cava. The left side of the heart was vented through PFO. Two everting 5-0 prolene stay sutures were placed and hanged on the left sternal blade. Working through the tricuspid valve, Langenbeck retractor is used to visualise the obstructive bands in RVOT. After initial resection of visible bands, we assess the residual RVOT by Jhajhria Infundibular Resection Adequacy Assessment Technique (JIRAAT).

In this technique, index finger of right hand is inserted into RVOT and the thumb of the same hand is placed on anterior aspect of RV. The residual muscle bundles are then assessed from above downwards from the pulmonary valve annulus towards the base of anterior tricuspid leaflet between the pulps of index finger and thumb. Then with the same fingers whole of the anterolateral RVOT assessed. Any residual muscle bundle, if felt can be visualised by retracting the tricuspid leaflets and resected under vision. Care should be taken to avoid cutting the papillary muscle and tricuspid chordae. This technique helps to avoid over resection and perforation of anterior RV wall as thickness of residual RVOT can be assessed precisely between the fingers (Image 1).

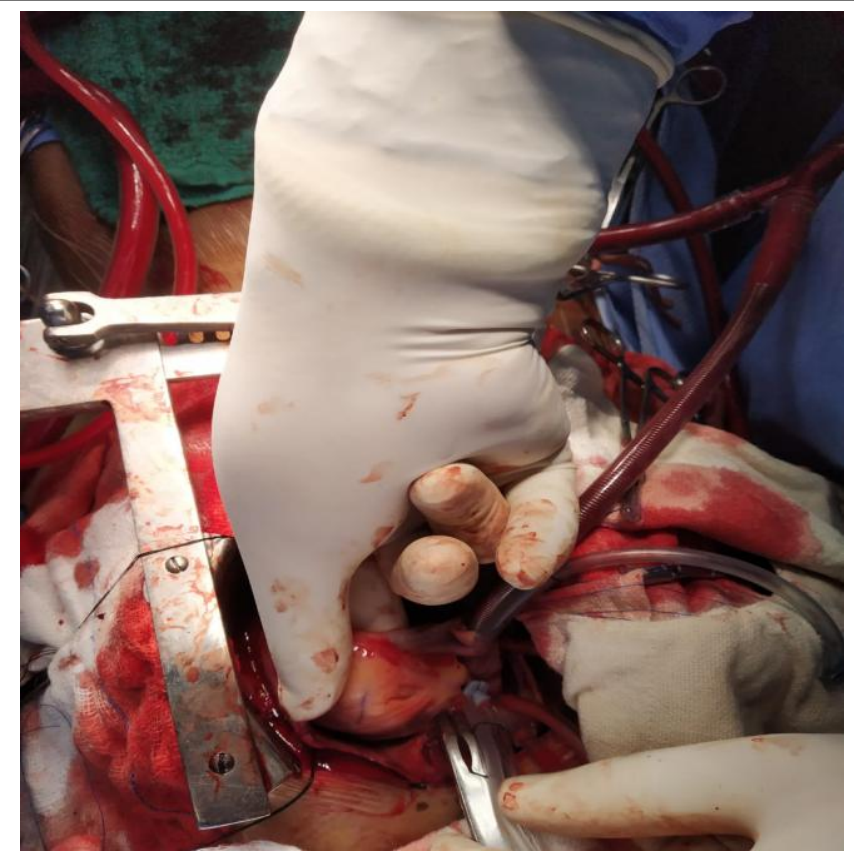

Image 1 demonstrating our technique of assessment of RVOT after resection with index finger inside the RVOT and thumb of right hand on outer surface

The VSD is then closed transatrially using interrupted prolene sutures and a tailored dacron patch. The tricuspid valve is assessed for competence and any distortion created by the VSD patch. Pulmonary annulus is then addressed. In our study, mean cross clamp time (CCT) was 77 minutes with shortest and longest CCT of 44 minutes and 122 minutes, respectively. Echocardiography before discharge did not reveal significant residual VSD in any patient. The mean RVOT pressure gradient was $28 \pm 5 \mathrm{mmHg}$. With this technique so far in 138 patients with none of the patients required reinstitution of $\mathrm{CPB}$ for residual RVOT obstruction because of inadequate resection.

\section{Discussion}

RVOT muscle resection is required in cases of for TOF, DCRV, DORV, VSD with Infundibular PS and other cases of infundibular muscle hypertrophy producing turbulence and obstruction in RVOT. Residual RVOT obstruction is an important factor that affects the outcome of corrective surgery 
Koushal et $\mathrm{al}^{[4]}$ described $35 \%$ incidence of significant RVOT obstruction after surgery, $12 \%$ of whom underwent immediate surgical revision. Besides immediate surgery, other patients need higher ionotropic supports to wean off cardiopulmonary bypass (CPB) in view of poor hemodynamis and RV dysfunction. It may require reinintervention within first $24 \mathrm{hrs}$ after surgery with resection / placing an RVOT patch. Later in postoperative patients residual gradients may lead to non regression of $\mathrm{RV}$ muscle mass contributing to arrhythmias and RV dysfunction.

Inadequate RVOT resection may be detected by assessing the RV pressures by inserting a needle connected to a pressure transducer sequentially in RV body, RVOT and MPA after weaning from CPB. Other techniques for detecting infundibular obstruction could be intraoperative Transesophageal echocardiography (TEE) or postoperative TTE.

One of the main reasons for residual obstruction due to muscle bundles is because of poor visibility of anterolateral muscle bundles hidden by the tricuspid leaflets and highly variable anatomy of the obstructing muscle bands in the form of their level, location and degree. With our technique, these muscle bands can be palpated and then resected, leaving very low incidence of residual RVOT obstructions. Alternatively, some authors detach the anterior tricuspid leaflet from annulus for better exposure to resect these bands ${ }^{[5]}$.

We have used our technique so far in more than 138 patients with none of the patients requiring reinstitution of $\mathrm{CPB}$ for residual RVOT obstruction because of inadequate resection. This technique does not prolong the aortic cross- clamp or CPB times and is reproducible, safe, and can be disseminated to junior colleagues and resident doctors.

This technique is very simple, safe, atraumatic and does not require any instrumentation. It may be very useful in isolation or as an adjunct to other methods for RVOT assessment like RV pressure, TEE, Echocardiography and Hegar sizing.
Funding: No funding sources

Conflict of interest: None declared

\section{References}

1. Dietl CA, Cazzaniga ME et al. Life threatening arrhythmias and RV dysfunction after surgical repair of tetrology of Fallot. Circulation 1994;5:711.

2. Stirling GR, Lillehei CW et al. The effects of cardiac bypass and ventriculomy upon right ventricular function. Surg Forum 1957;8:433- 438.

3. Hudspeth AS, Cordell AR et al. Transatrial approach to total correction of Tetralogy of Fallot. Circulation 1963; 27:796-800.

4. Koushal SK, Iyer KS et al. Significant intraoperative right ventricular outflow gradients after repair of tetrology of fallot: To revise or not to revise? Annals of thoracic surgery 1999;68:1705-1713.

5. Koshy S, Sunil GS, Anil SR et al. Tricuspid valve detachment for transatrial closure of ventricular septal defects. Asian Cardiovasc Thorac Ann 2002; 10 (4): 314 -7 . 